\title{
Quantum Zakharov Equations
}

\author{
Fernando Haas ${ }^{* \dagger}$ \\ UNISINOS, Rio Grande do Sul, Brazil \\ E-mail: ferhaas@exatas.unisinos.br \\ Leonardo Geissler Garcia \\ UNISINOS, Rio Grande do Sul, Brazil \\ E-mail: garcia@exatas.unisinos.br \\ João Goedert \\ UNISINOS, Rio Grande do Sul, Brazil \\ E-mail: goedert@exatas .unisinos.br
}

In this paper we investigate the existence of soliton solutions for a modified form of the Zakharov equations describing modulational instabilities in quantum plasmas. In particular, we show that quantum effects suppress the easily identifiable soliton solutions, obtained in the adiabatic limit. In this limit, the quantum Zakharov equations become a coupled fourth order system, not amenable to straightforward integration as it was the case for the integrable nonlinear Schrödinger equation. By considering the simultaneous adiabatic and semiclassical limits, we obtain more detailed results through a variational solution. Specifically these results show that quantum effects enhance the dispersion and smear out the classical one soliton solution.

Fourth International Winter Conference on Mathematical Methods in Physics 09 - 13 August 2004

Centro Brasileiro de Pesquisas Físicas (CBPF/MCT), Rio de Janeiro, Brazil

\footnotetext{
* Speaker.

${ }^{\dagger}$ We thanks the Brazilian agency Conselho Nacional de Desenvolvimento Científico e Tecnólogico (CNPq) for financial support.
} 


\section{Introduction}

There has been increased interest on the relevance of quantum effects on plasmas under extreme conditions such as those encountered in laser and some astrophysical plasmas or in ultra small electronic devices [1]-[5]. Quantum plasmas are very complicated and the difficulty to develop and explore full fledged quantum descriptions has motivated the introduction of approximated models to describe plasmas with quantum corrections. One of these approaches is the multistream quantum model proposed by Haas et al. [1] and recently generalized by Anderson et al. [2] who incorporated statistical effects. Applied to a two stream system, the multistream quantum plasma model predicts some new instabilities (attenuated by statistics, [2]) and provides an overall stabilization mechanism for large wave numbers. The same dispersive and stabilizing contribution appears for quantum effects in three stream plasmas [3]. Similar behavior also occurs for quantum plasmas described within the framework of the Wigner-Poisson system [4, 5].

In classical plasmas, nonlinear phenomena are frequently formulated in terms of completely integrable evolution equations of the Korteweg-de Vries (KdV) or nonlinear Schrödinger equation (NLS) type [6]-[8]. These completely integrable equations, as is well known, admit N-soliton solutions, derivable from the Inverse Scattering Transform (IST) method. Soliton solutions follow from a delicate balance between nonlinearity and dispersion, and a natural question concerns the role quantum effects play in this regard. More exactly, how do quantum effects alter soliton formation in plasmas?

We will try to answer such questions by applying the same weak nonlinearity expansion, used to study classical plasmas, on the quantum hydrodynamic model [9]. This procedure yields a modified $\mathrm{KdV}$ equation [10] that describes the evolution of ion acoustic waves. As shown in [10], quantum effects can distort or even suppress the usual one soliton solutions, as a result of the quantum dispersion effects. Following this line, the interaction between ion acoustic and Langmuir waves in quantum plasmas has been studied [11], in this case in the framework of Zakharov equations [6]-[8] with quantum corrections. The linear modes of this system [11] were shown to have stronger stability properties in comparison with the classical plasma case. Nonlinear waves for the quantum Zakharov equations, however, are not yet sufficiently well understood. For instance, the classical Zakharov equations in the adiabatic limit reduce to the NLS equation which is completely integrable in terms of the IST method. No similar technique seems to be easily constructed in the quantum case.

The purpose of this study is to investigate the role played by quantum effects in the nonlinearity/dispersion balance leading to the formation of soliton solutions for the Zakharov equations. This is more easily done in the context of a variational formulation derived for the simultaneous adiabatic and semiclassical regimes of the quantum Zakharov equations. In particular we show that in this situation the envelope of the high frequency electric field of the quantum plasma is described by a decoupled nonlinear evolution equation. In the next section, we derive this modified equation which, in the formal classical limit, becomes the NLS equation. In terms of the variational formulation, we propose a variational solution reproducing the NLS one-soliton. In the sequence we investigate the properties of the variational solution. 


\section{Zakharov equations with a quantum correction}

Consider a two species plasma composed of electrons and ions, the ions being much more massive than electrons. In this system, we identify two different time scales, the slow time scale of the ions and the fast time scale of the electrons. This suggests a development in terms of two time scales. It is the low mobility of the ions compared to that of the electrons that justifies this kind of treatment. Starting with the quantum hydrodynamic model for plasmas with a quantum correction $[9,10]$ and adopting the same procedure used [6]-[8] in the classical approach, we obtain the following modified quantum Zakharov equations, in one spatial variable

$$
\begin{aligned}
& i \frac{\partial E}{\partial t}+\frac{\partial^{2} E}{\partial x^{2}}-H^{2} \frac{\partial^{4} E}{\partial x^{4}}=n E, \\
& \frac{\partial^{2} n}{\partial t^{2}}-\frac{\partial^{2} n}{\partial x^{2}}+H^{2} \frac{\partial^{4} n}{\partial x^{4}}=\frac{\partial^{2}|E|^{2}}{\partial x^{2}} .
\end{aligned}
$$

In these equations, non-dimensional variables are used throughout, $E$ is the envelope of an electric field, and $n$ is the plasma density deviation measured from its equilibrium value. In addition, we introduce

$$
H=\frac{\hbar \omega_{i}}{\kappa_{B} T_{e}}
$$

a parameter that measures the importance of the quantum effects in modulational instabilities. In this definition, $\omega_{i}$ is the ion plasma frequency and $T_{e}$ the temperature of the electron fluid. Notice that the quantum parameter $H$ is the ratio of an ionic term, the ion plasmon energy, and an electronic term, the electron kinetic energy. In fact, the coupling between inertial (ionic) and dynamic (electronic) terms is characteristic of phenomena involving the propagation of ion acoustic waves in plasmas. This is the case for the ion acoustic wave velocity $c_{s}=\left(\kappa_{B} T_{e} / m_{i}\right)^{1 / 2}$, where $m_{i}$ is the ion mass. In the formal classical limit $H \rightarrow 0$, the quantum Zakharov equations recover the classical [6]-[8] Zakharov system. For the details on the derivation of (2.1-2.2), we refer to [11].

Solutions of the classical $(H=0)$ Zakharov system are found, in the adiabatic limit, by setting $\partial^{2} n / \partial t^{2} \approx 0$ in (2.2). In this situation, the envelope of the electric field satisfies a nonlinear Schrödinger equation, which is completely integrable yielding $\mathrm{N}$-soliton solutions. An interesting question at this point is if the quantum effects may perturb or perhaps even destroy these localized solitonic solutions. Indeed, solitons usually arise as a consequence of the detailed balance between dispersive and nonlinear contributions. Since quantum effects enhance dispersion, one should expect that quantum solitons will not be so easily found for quantum Zakharov equations as for the classical case. Let us investigate this conjecture by taking $\partial^{2} n / \partial t^{2} \approx 0$ in (2.2). Integrating twice and assuming decaying boundary conditions, immediately gives

$$
n=-|E|^{2}+H^{2} \frac{\partial^{2} n}{\partial x^{2}}
$$

Equation (2.4), inserted in Eq. (2.1), yields

$$
i \frac{\partial E}{\partial t}+\frac{\partial^{2} E}{\partial x^{2}}+|E|^{2} E=H^{2}\left(\frac{\partial^{4} E}{\partial x^{4}}+E \frac{\partial^{2} n}{\partial x^{2}}\right) .
$$


In the formal classical limit $H \rightarrow 0$, the right hand side of Eq. (2.5) vanishes and the completely integrable NLS equation are recovered. However, for $H \neq 0$ the traditional reduction procedure of searching for solutions in the form

$$
E=F(x-M t) \exp (i[k(x-u t)+\delta]), \quad n=G(x-M t),
$$

for real functions $F$ and $G$, and parameters $k, M, u$, and $\delta$, does not seem to produce any results here. The basic difficulty stems from the fact that the new equations constitute a complicated fourth order system of coupled, nonlinear equations. The existence of localized or soliton solutions for this new system remains an open question. However, some insight on this issue can be gained by considering the simultaneous adiabatic and semiclassical limits. Substituting (2.4) into (2.5) and retaining only terms of order up to $H^{2}$, yields the decoupled equation

$$
i \frac{\partial E}{\partial t}+\frac{\partial^{2} E}{\partial x^{2}}+|E|^{2} E=H^{2}\left(\frac{\partial^{4} E}{\partial x^{4}}-E \frac{\partial^{2}|E|^{2}}{\partial x^{2}}\right) .
$$

Equation (2.7) is derivable from a variational principle,

$$
\delta S=\delta \int \mathcal{L} d x d t=0
$$

based on the Lagrangian density

$$
\mathcal{L}=\frac{i}{2}\left(E \frac{\partial E^{*}}{\partial t}-E^{*} \frac{\partial E}{\partial t}\right)+\frac{\partial E}{\partial x} \frac{\partial E^{*}}{\partial x}-\frac{|E|^{4}}{2}+H^{2} \frac{\partial^{2} E}{\partial x^{2}} \frac{\partial^{2} E^{*}}{\partial x^{2}}-\frac{H^{2}}{2}|E|^{2} \frac{\partial^{2}|E|^{2}}{\partial x^{2}} .
$$

It is now ease to check that the variational derivatives $\delta S / \delta E^{*}=\delta S / \delta E=0$ produce (2.7) and its complex conjugate equation, respectively.

Inspired on the form of the classical soliton solutions, we proceed by proposing time-dependent variational solutions

$$
E=\alpha(t) \exp (i \theta(t)) \operatorname{sech}(\beta(t) x),
$$

dependent on adjustable real $\alpha, \beta$, and $\theta$, considered as functions of time only. This form warrants that the classical one soliton solutions for the nonlinear Schrödinger equation are recovered whenever $\alpha=\sqrt{2 \Omega}, \theta=\Omega t$, and $\beta=\sqrt{\Omega}$, for $\Omega$ constant. After inserting (2.10) into (2.8), we get a mechanical system governed by the action

$$
S=\int L d t
$$

constructed from the Lagrange

$$
L=L(\theta, \alpha, \beta, \dot{\theta}, \dot{\alpha}, \dot{\beta})=\frac{\alpha^{2}}{15}\left(\frac{30 \dot{\theta}}{\beta}-\frac{10 \alpha^{2}}{\beta}+10 \beta+8 H^{2} \alpha^{2} \beta+14 H^{2} \beta^{3}\right)
$$

We can now compute the variational derivatives and obtain

$$
\begin{aligned}
& \frac{\delta S}{\delta \theta}=0 \quad \Rightarrow \quad \frac{d}{d t}\left(\frac{\alpha^{2}}{\beta}\right)=0 \\
& \frac{\delta S}{\delta \alpha}=0 \quad \Rightarrow \quad \alpha\left(\dot{\theta}-\frac{2 \alpha^{2}}{3}+\frac{\beta^{2}}{3}+\frac{8 H^{2} \alpha^{2} \beta^{2}}{15}+\frac{7 H^{2} \beta^{4}}{15}\right)=0 \\
& \frac{\delta S}{\delta \beta}=0 \quad \Rightarrow \quad \alpha^{2}\left(\dot{\theta}-\frac{\alpha^{2}}{3}-\frac{\beta^{2}}{3}-\frac{4 H^{2} \alpha^{2} \beta^{2}}{15}-\frac{7 H^{2} \beta^{4}}{5}\right)=0 .
\end{aligned}
$$


Equation (2.13) has a simple solution

$$
\alpha^{2}=2 \sqrt{\Omega} \beta
$$

for $\Omega$ a numerical constant. By discarding the trivial case $\alpha=0$ and inserting (2.16) into (2.14), we derive

$$
\dot{\theta}=\frac{4 \sqrt{\Omega} \beta}{3}-\frac{\beta^{2}}{3}-\frac{16 \sqrt{\Omega} H^{2} \beta^{3}}{15}-\frac{7 H^{2} \beta^{4}}{15}
$$

which, after taking into account (2.15), yields

$$
H^{2} \beta^{3}+\frac{6 \sqrt{\Omega} H^{2} \beta^{2}}{7}+\frac{5 \beta}{14}-\frac{5 \sqrt{\Omega}}{14}=0 .
$$

Equation (2.18) is the key equation for our variational treatment to the semiclassical Zakharov system in the adiabatic limit. A useful simplification is achieved by adopting the rescaling

$$
\bar{\beta}=\frac{\beta}{\sqrt{\Omega}}, \quad \bar{H}=\sqrt{\Omega} H,
$$

which eliminates one irrelevant parameter in Eq. (2.18),

$$
\bar{H}^{2} \bar{\beta}^{3}+\frac{6 \bar{H}^{2} \bar{\beta}^{2}}{7}+\frac{5 \bar{\beta}}{14}-\frac{5}{14}=0 .
$$

In the formal classical limit $\bar{H}=0$, we obtain $\bar{\beta}=1$, which reproduces the classical one soliton solution. In addition, it can be shown that, for

$$
\bar{H}^{2} \leq \frac{5}{1152}(681+23 \sqrt{897}) \approx 5.946,
$$

Eq. (2.20) admits just one real root besides two complex conjugate ones. This range of parameters seems to be in accordance with the semiclassical limit we have taken. However, it is important to notice that, in view of the dependence of $\bar{H}$ on $\Omega$ (see Eq. (2.19)), no constraint is imposed on the maximum value of $\bar{H}$, provided $H \neq 0$, and sufficiently high values of $\Omega$ are chosen. Hence, we now consider both cases separately.

\subsection{Small $\bar{H}^{2}$}

In this situation, Eq. (2.20) possesses the perturbative solution

$$
\bar{\beta}=1-\frac{26 \bar{H}^{2}}{5}+O\left(\bar{H}^{4}\right)
$$

By retaining only terms of order up to $\bar{H}^{2}$ and going back to the original variables, we get

$$
\begin{aligned}
& \alpha=\sqrt{2 \Omega}\left(1-\frac{13 \Omega H^{2}}{5}\right) \\
& \beta=\sqrt{\Omega}\left(1-\frac{26 \Omega H^{2}}{5}\right) \\
& \dot{\theta}=\Omega-5 \Omega^{2} H^{2}
\end{aligned}
$$


Inspection of the variational solution (2.10), leads to the conclusion that the quantum effects contribute, in the small $\bar{H}$ limit, to decrease the amplitude (proportional to $\alpha$ ), enlarge the spatial dispersion (proportional to $1 / \beta$ ), and to decrease the rate of change of the phase (proportional to $\dot{\theta}$ ) of the classical soliton. All this is in accordance with the dispersive character of quantum effects in the semiclassical limit. They contribute to smear the soliton out, perturbing (perhaps in a crucial way) the critical balance between the nonlinearity and dispersion effects which supported the existence of the soliton solutions for the nonlinear Schrödinger equation.

\subsection{Large $\bar{H}^{2}$}

We can access $\bar{H}=\sqrt{\Omega} H \gg 1$, even for the semiclassical $H \ll 1$ case we are considering, provided $H \neq 0$ and $\Omega$ is taken sufficiently large. For large $\bar{H}$ we find, instead of just one class of solutions as in the preceding subsection, three different subclasses of solutions, namely

$$
\begin{aligned}
& \bar{\beta}_{0}=-\frac{6}{7}+\frac{65}{72} \bar{H}^{-2}+O\left(\bar{H}^{-4}\right) \\
& \bar{\beta}_{+}=\left(\frac{5}{12}\right)^{1 / 2} \bar{H}^{-1}-\frac{65}{144} \bar{H}^{-2}+O\left(\bar{H}^{-3}\right), \\
& \bar{\beta}_{-}=-\left(\frac{5}{12}\right)^{1 / 2} \bar{H}^{-1}-\frac{65}{144} \bar{H}^{-2}+O\left(\bar{H}^{-3}\right) .
\end{aligned}
$$

It is important to mention that the first solution comes from an expansion in $1 / \bar{H}^{2}$, while the last two solutions result from an expansion in $1 / \bar{H}$. As a consequence, the accuracy of these perturbative solutions are different as can be easily checked by taking some fixed large value of $\bar{H}^{2}$, solving (2.20) and comparing with (2.26-2.27).

In view of (2.16), a purely imaginary solution $\alpha$ is obtained for Eq. (2.26), when the solution of Eq. (2.28) with the negative sign is used and only leading order terms are retained in the expansion. This is in contradiction with the proposal (2.10), constructed with real functions $\alpha, \beta$ and $\theta$. Hence we discard these solutions and consider only $\bar{\beta}_{+}$in (2.27). This yields, again considering only leading order terms,

$$
\alpha=\left(\frac{5 \Omega}{3 H^{2}}\right)^{1 / 4}, \quad \beta=\frac{(5 / 12)^{1 / 2}}{H}, \quad \dot{\theta}=\frac{4}{9} \sqrt{\frac{5 \Omega}{3 H^{2}}} .
$$

Since $H \ll 1$ and $\Omega \gg 1$, this corresponds to a large amplitude, highly localized, and highly oscillating variational solution, which has no classical correspondence.

\section{Conclusion}

Using a variational principle, we show that quantum effects tend to smear out the one soliton solutions of the quantum Zakharov equations, in the adiabatic semiclassical limit. This is not an obvious result, specially if we realize that quantum terms appear also in a nonlinear way in the right hand side of equation (2.7). In addition, we have found a new variational solution, with no classical counterpart. It must be emphasized though, that the nonlinear wave solutions for the quantum Zakharov equations are still only poorly understood. For instance, an important open question concerns the influence of quantum effects in $\mathrm{N}$-soliton solutions and their stability. In the classical 
case, numerical experiments [6]-[8] typically show that the solutions for the Zakharov equations relax asymptotically to $\mathrm{N}$-soliton solutions. To this moment, no similar numerical investigation has been reported, for the correponding quantum Zakharov equations.

\section{References}

[1] F. Haas, G. Manfredi, and M. R. Feix, Multistream model for quantum plasmas, Phys. Rev. E62 (2000) 2763.

[2] D. Anderson, B. Hall, M. Lisak, and M. Marklund, Statistical effects in the multistream model for quantum plasmas, Phys. Rev. $\mathbf{E 6 5}$ (2002) 046417.

[3] F. Haas, G. Manfredi, and J. Goedert, Stability analysis of a three-stream quantum plasma equilibrium, Braz. J. Phys. 33 (2003) 128.

[4] F. Haas, G. Manfredi, and J. Goedert, Nyquist method for Wigner-Poisson quantum plasmas, Phys. Rev. E64 (2001) 26413.

[5] A. Luque, H. Schamel, and R. Fedele, Quantum corrected electron holes, Phys. Lett. A324 (2004) 185.

[6] M. V. Goldman, Strong turbulence of plasma waves, Rev. Mod. Phys. 56 (1984) 709.

[7] V. E. Zakharov, Collapse of Langmuir waves, Sov. Phys. JETP 35 (1972) 908.

[8] S. G. Thornhill and D. ter Haar, Langmuir turbulence and modulational instability, Phys. Rep. 43 (1978) 43.

[9] G. Manfredi and F. Haas, Self-consistent fluid model for a quantum electron gas, Phys. Rev. B64 (2001) 075316.

[10] F. Haas, L. G. Garcia, J. Goedert, and G. Manfredi, Quantum ion-acoustic waves, Phys. Plasmas 10 (2003) 3858.

[11] L. G. Garcia, F. Haas, L. P. L. de Oliveira and J. Goedert, Modified Zakharov equations for plasmas with a quantum correction, physics/0410257. 